\title{
NONEXPANSIVE ACTIONS OF TOPOLOGICAL SEMIGROUPS ON STRICTLY CONVEX BANACH SPACES AND FIXED POINTS
}

\author{
WOJCIECH BARTOSZEK
}

(Communicated by John B. Conway)

\begin{abstract}
Let $C$ be a closed convex subset of a strictly convex Banach space $X$ and $\left\{T_{s}: s \in S\right\}$ be a continuous representation of a semitopological semigroup $S$ as nonexpansive mappings of $C$ into itself. The main result establishes the fact that if for some $x \in C$ the trajectory $\left\{T_{s} x: s \in S\right\}$ is relatively compact and $A P(S)$ has a left invariant mean then $K=\overline{\operatorname{conv}\left\{T_{s} x: s \in S\right\}}$ contains a common fixed point for $\left\{T_{s}\right\}_{s \in S}$.
\end{abstract}

Let $C$ be a closed, convex subset of a Banach space $X$, and $T$ be a nonexpansive mapping of $C$ into $C$ (i.e., $\|T x-T y\| \leq\|x-y\|$ for every $x, y \in C$ ). For $x \in C$ the orbit of $x$ is the set $\mathscr{O}(x)=\left\{T^{n} x: n \geq 0\right\}$ and the $\omega$-limit set of $x$ is defined by $\omega(x)=\left\{y: \lim T^{n_{k}} x=y\right.$ for some subsequence $\left.n_{k} \nearrow \infty\right\}$. The $\omega$-limit set of a point $x$ is easily shown to be closed and $T$-invariant although possibly empty. If $\omega(x)$ is nonempty then it is minimal (the orbit $\mathscr{O}(y)$ is a dense subset of $\omega(x)$ for every $y \in \omega(x)$ ) and the action of $T$ on $\omega(x)$ is isometric (see [D.S.]). By [R.S.], nonempty $\omega(x)$ can be given the structure of a monothetic group. So there exists a $T$-invariant probability measure $\mu\left(\mu\right.$ is invariant if $\left.\mu \circ T^{-1}=\mu\right)$ on $\omega(x)$ if and only if $\omega(x)$ is (nonempty) compact (see also [B.D.]). The following lemma gives connections between the existence of compact orbits and $T$-invariant measures in slightly more general situations.

LEMMA 1. Let $X, C, T$ be as above. If $C$ is separable and $\mu$ is a $T$-invariant probability (on a Borel $\sigma$-field) then every $x \in \operatorname{supp} \mu$ is recurrent and $\omega(x)$ is compact (supp $\mu$ denotes here the smallest closed subset of $C$ of full measure $\mu$ ).

Proof. Notice that supp $\mu$ is a $T$-invariant subset of $C$ (i.e., if $x \in \operatorname{supp} \mu$ then $T x \in \operatorname{supp} \mu)$. By the classical Poincare recurrence theorem, the set of recurrent points is full measure, so the set of recurrent points is dense in $\operatorname{supp} \mu$. Since $T$ is nonexpansive, every $x \in \operatorname{supp} \mu$ is recurrent. Thus $\operatorname{supp} \mu=\bigcup_{x \in \operatorname{supp} \mu} \omega(x)$, and for every $x, y \in \operatorname{supp} \mu$ the limit sets $\omega(x), \omega(y)$ coincide or are disjoint. In order to show compactness of $\omega(x)$ it is enough to show the existence of $T$-invariant probability on $\omega(x)$. Let $\mathscr{T}$ denote the partition of $W=\operatorname{supp} \mu$ on the sets $\omega(x)$. It is known (see $[\mathbf{R}]$ or $[\mathbf{P}]$ ) that there exists a system of canonical measures $\mu_{\tau}$ concentrated on $\tau(\tau=\omega(x)$ for $x \in W)$ such that

$$
\mu=\int_{W / \mathscr{G}} \mu_{\tau} \nu(d \tau)
$$

Received by the editors January 30, 1987 and, in revised form, November 30, 1987.

1980 Mathematics Subject Classification (1985 Revision). Primary 47H10, 47H20; Secondary 47H09. 
and $\nu$ is some probability measure on $W / \mathscr{T}$. Let $\sigma_{\mathscr{T}}$ denote the sub $\sigma$-field generated by sets of the partition $\mathscr{T}$. Clearly

$$
\begin{aligned}
\int f d \mu_{\omega(x)} & =E\left(f \mid \sigma_{\mathscr{T}}\right)(x)=E\left(f \mid \sigma_{\mathscr{G}}\right)(T x) \\
& =E\left(f \circ T \mid \sigma_{\mathscr{T}}\right)(x)=\int f \circ T d \mu_{\omega(x)},
\end{aligned}
$$

for $\nu$ almost all $\omega(x) \in W / \mathscr{T}(f$ is here an arbitrary continuous function on supp $\mu$ ). Thus, for $\nu$ and almost all $\tau$, the measures $\mu_{\tau}$ are $T$-invariant, so $\omega(x)$ is compact for $x$ from a dense subset of $W$ (see [R.S.] or [B.D.]). But the set $\{y: \omega(y)$ is compact $\}$ is closed (and convex if $X$ is strictly convex), and thus for all $x \in \operatorname{supp} \mu$ the orbit $\omega(x)=\overline{\mathscr{O}(x)}$ is compact.

Proposition. Let $X$ be a strictly convex Banach space and $C$ be a separable, convex, closed subset of $X$. If $T: C \rightarrow C$ is nonexpansive then the following conditions are equivalent:

(i) the set $F(T)$ of fixed points of $T$ is nonempty;

(ii) there exists a $T$-invariant probability;

(iii) there exists $x \in C$ such that $\overline{\mathscr{O}(x)}$ is compact.

Proof. Because (i) $\Rightarrow$ (ii) and (iii) $\Rightarrow$ (ii) are trivial and (ii) $\Rightarrow$ (iii) follows from our lemma we only have to show (ii) $\Rightarrow(\mathrm{i})$. Let $\mu$ be the $T$-invariant probability measure on $\omega(x)$ (unique by nonexpansiveness of $T$ ). Since $T$ is affine on $\overline{\operatorname{conv} \omega(x)}$ (see $[\mathbf{E}]$ or $[\mathbf{Y}]$ ), for every $x^{*} \in X^{*}, x \circ T$ is affine, continuous and $x^{*}(\operatorname{bar} \mu)=$ $\int_{\omega(x)} x^{*}(y) \mu(d y)=\int_{\omega(x)} x^{*} \circ T(y) \mu(d y)=x^{*} \circ T(\operatorname{bar} \mu)=x^{*}(T(\operatorname{bar} \mu))$. Since the functionals separate points of $X$ the barycenter of $\mu$ is a fixed point of $T$.

Let $S$ be a semitopological semigroup, i.e., $S$ is a semigroup with a Hausdorff topology such that for each $a \in S$ the mappings $s \rightarrow a s$ and $s \rightarrow s a$ from $S$ to $S$ are continuous. Let $X$ be a strictly convex Banach space, and $S \ni s \rightarrow T_{s}$ be a continuous representation of $S$ as nonexpansive mappings on a closed convex subset $C$ of $X$ into $C$, i.e., $T_{a b}(x)=T_{a}\left(T_{b}(x)\right), a, b \in S, x \in C$, and the mapping $s \rightarrow T_{s} x$ from $S$ into $C$ is continuous for every $x \in C$. If $f \in C(S)$ and $a \in S$ define $l_{a} f(s)=f(a s)$ and $r_{a} f(s)$ for all $s \in S(C(S)$ denotes here the set of all continuous bounded functions on $S)$. Recall, a function $f \in C(S)$ is said to be almost periodic on $S$ if $\left\{r_{a} f: a \in S\right\}$ is relatively compact in the norm topology of $C(S)$. The subalgebra of all almost periodic functions on $S$ we denote by $A P(S)$. A linear functional $m \in A P(S)^{*}$ is called a left invariant mean if for all $a \in S$ and $f \in A P(S)$ we have $\left\langle l_{a} f, m\right\rangle=\langle f, m\rangle$ and $\langle 1, m\rangle=1$. The following theorem is a partial solution of Problem 2 from [L.2].

THEOREM. Let $\left\{T_{s}: s \in S\right\}$ be a continuous representation of a semitopological semigroup $S$ as nonexpansive mappings on a closed convex subset of a strictly convex Banach space $X$. If $A P(S)$ has a left invariant mean, $x \in C$ such that $\left\{T_{s} x: s \in S\right\}$ is relatively compact then $\left.K=\overline{\operatorname{conv}\left\{T_{s} x: s \in S\right.}\right\}$ contains a common fixed point for $\left\{T_{s}\right\}_{s \in S}$.

Proof. It is clear that for every continuous function $f$ on $C$ the function $\tilde{f}$ defined on $S$ as $\tilde{f}(s)=f\left(T_{s} x\right)$ belongs to $A P(S)$. Thus a left invariant mean $m$ on $A P(S)$ defines a probability measure $\mu$ on $\overline{\left\{T_{s} x: s \in S\right.}$. Clearly (see [L.1]), the measure $\mu$ 
is $T_{s}$-invariant for every $s \in S$. By Lemma 1 , if $y \in \operatorname{supp} \mu$ then for every $s \in S, y$ is $T_{s}$ recurrent and $T_{s}$ is affine on $K^{\prime}=\overline{\operatorname{conv}(\operatorname{supp} \mu)} \subseteq K$. But by our proposition the barycenter of measure $\operatorname{bar}(\mu) \in K^{\prime}$ is a fixed point for every $T_{s}$.

It is a pleasure to thank Professors Lau and Sine for sending me preprints of some of their works. These lead to the following remark: Problem 2 of Lau [L.2] has a negative answer in general. There is an appropriate nonexpansive map $T$ in a 3-dimensional (Banach) space for which $(N+1)^{-1}\left(I+T+\cdots+T^{N}\right) x$ converges, but the limit is not a fixed point, and there is no fixed point in the closed convex hull of the orbit (see Robert Sine, Behaviour of iterates in the Poincare metric, preprint, 1986).

\section{REFERENCES}

[B.D.] W. Bartoszek and T. Downarowicz, Compactness of trajectories of dynamical systems in uniform complete spaces, Suppl. Rend. Circ. Mat. Palermo (The 13th Winter School on Abstract Analysis, Srni, 1985).

[D.S.] C. M. Dafermos and M. Slemrod, Asymptotic behaviour of nonlinear contraction semigroup, J. Funct. Anal. 13 (1973), 97-106.

[E] M. Edelstein, On non-expansive mappings of Banach spaces, Proc. Cambridge Philos. Soc. 60 (1964), 439-447.

[L.1] A. T. Lau, Invariant means on almost periodic functions and fixed point properties, Rocky Mountain J. Math. 3 (1973), 69-76.

[L.2] A. T. Lau, Semigroup of nonexpansive mappings on a Hilbert space, J. Math. Anal. Appl. 105 (1985), 514-522.

[P] W. Parry, Entropy and generators in ergodic theory, Yale Univ. Press, New Haven, Connecticut, 1966.

[R] W. A. Rochlin, Isbrannyje woprosy mietriczieskoj teorii dynamichieskich sistiem, Uspekhi Mat. Nauk 30 (1949), 57-128.

[R.S.] S. Roehring and R. C. Sine, The structure of $\omega$-limit sets of nonexpansive maps, Proc. Amer. Math. Soc. 81 (1981), 398-400.

[Y] C-L. Yen, On the rest points of a nonlinear nonexpansive semigroup, Pacific J. Math. 45 (1973), 699-706.

Institute of Mathematics, Polish academy of Sciences, Wroclaw Branch, KOPERNIKA 18, 51-617 WROClAW, POLAND

Current address: ul. Sułkowskiego 4 m. 14, 85-655 Bydgoszcz, Poland 